\title{
A Box-Consistency Contractor Based on Extremal Functions
}

\author{
Gilles Trombettoni, Yves Papegay, Gilles Chabert, Odile Pourtallier \\ COPRIN INRIA, Université Nice-Sophia, LINA Ecole des Mines de Nantes \\ \{Gilles.Trombettoni, Yves.Papegay, Odile.Pourtallier\}@sophia.inria.fr, \\ Gilles.Chabert@emn.fr
}

\begin{abstract}
Interval-based methods can approximate all the real solutions of a system of equations and inequalities. The Box interval constraint propagation algorithm enforces Box consistency. Its main procedure BoxNarrow handles one function $f$ corresponding to the revised constraint, and one variable $x$, replacing the other variables of $f$ by their current intervals. This paper proposes an improved BoxNarrow procedure for narrowing the domain of $x$ when $f$ respects certain conditions. In particular, these conditions are fulfilled when $f$ is polynomial. $f$ is first symbolically rewritten into a new form $g$. A narrowing step is then run on the non-interval extremal functions that enclose the interval function $g$. The corresponding algorithm is described and validated on several numerical constraint systems.
\end{abstract}

\section{Motivation}

Interval-based solvers can solve systems of numerical constraints (i.e., nonlinear equations or inequalities over the reals). Their reliability and increasing performance make them applicable to various domains such as robotics design and kinematics [9], proofs of conjectures [12], robust global optimization [7,11] and bounded-error parameter estimation [6].

Two main types of contraction algorithms allow solvers to filter variable domains, i.e., to reduce the intervals of each variable, without loss of solutions of the system: interval (numerical) analysis methods, like Interval Newton [10], and constraint propagation algorithms from constraint programming. The HC4 and Box algorithms $[2,14]$ are very often used in solving strategies. They perform a propagation loop and filter the variable domains with a specific revise procedure (called HC4-Revise and BoxNarrow) handling the constraints individually. For every pair $(c, x)$ in the system, where $c$ is the numerical constraint $f\left(x, y_{1}, \ldots, y_{k-1}\right)=0$, the BoxNarrow contraction procedure is applied to $x$ by considering the uni-variate constraint: $f_{[Y]}(x)=f\left(x,\left[y_{1}\right], \ldots,\left[y_{k-1}\right]\right)=0$. That is, $f_{[Y]}$ is a function where each variable $y_{i} \in Y=\left\{y_{1}, \ldots, y_{k-1}\right\}$ of $f$ has been replaced by its interval of variation. The important point is that $f_{[Y]}$ is an interval function: to any $x \in \mathbb{R}, f_{[Y]}(x)$ is an interval. Thus, the iterative process run by BoxNarrow may be very slow in some cases. The main idea of PolyBox is to work with two non-interval functions instead of $f_{[Y]}$. These non-interval functions are obtained by a symbolic manipulation preprocessing that rewrites 
$f$ into a new form $g$ for which the two extremal functions enclosing $g_{[Y]}$ can be easily extracted. Then, during constraint propagation, PolyBoxRevise calls BoxNarrow on the two extremal functions of $g_{[Y]}$. This implies a faster contraction. In addition, when $g_{[Y]}$ is a low-degree polynomial, the computation of the new bounds of $[x]$ follows simple evaluations using the real roots of the extremal functions identified analytically.

\section{Background}

Intervals allow reliable computations on computers by managing floating-point bounds and outward rounding.

\section{Definition 1 (Basic definitions, notations)}

$\mathbb{I} \mathbb{R}$ denotes the set of intervals $[v]=[a, b] \subset \mathbb{R}$ where $a$, also denoted $\underline{v}$, and $b$, also denoted $\bar{v}$, are floating-point numbers. $\bar{v}-\underline{v}$ is the size of $[v]$.

An interval vector, or box, $[V]=\left(\left[v_{1}\right], \ldots,\left[v_{n}\right]\right)$ represents the Cartesian product $\left[v_{1}\right] \times \ldots \times\left[v_{n}\right]$. Its size is the maximal size of its components $\left[v_{i}\right], i=$ $1, \ldots, n$.

Interval arithmetic has been introduced to extend the real arithmetic to intervals [10]. For instance, we have straightforwardly $\left[v_{1}\right]+\left[v_{2}\right]=\left[\underline{v_{1}}+\underline{v_{2}}, \overline{v_{1}}+\overline{v_{2}}\right]$. This allows us to extend real valued functions to intervals. Such an extension must be defined so as to be conservative, i.e., $\forall V \in \mathbb{R}^{k} \quad f(V)=[f](V)$ and $\forall[V] \in \mathbb{R}^{k}[f]([V]) \supseteq\{f(V), V \in[V]\}$.

The natural extension $[f]_{N}$ of a real function $f$ replaces arithmetic over the reals by interval arithmetic. Consider for instance the real function $f\left(x_{1}, x_{2}\right)=$ $x_{1}^{2}-2 x_{1} x_{2}+x_{2}^{2}$. The natural extension $[f]_{N}$ from $\mathbb{R}^{n}$ to $\mathbb{R}$ is defined by $[f]_{N}\left(\left[x_{1}\right],\left[x_{2}\right]\right)=\left[x_{1}\right]^{2}-2\left[x_{1}\right]\left[x_{2}\right]+\left[x_{2}\right]^{2}$. Evaluated on the intervals $\left[x_{1}\right]=$ $\left[x_{2}\right]=[0,1]$, we obtain $[f]_{N}\left(\left[x_{1}\right],\left[x_{2}\right]\right)=[-2,2]$. Note that the natural extension of $f$ depends upon its symbolic expression, and consequently is not unique. As a matter of fact, $f$ may also be rewritten as $\left(x_{1}-x_{2}\right)^{2}$ and yields the natural extension $\left(\left[x_{1}\right]-\left[x_{2}\right]\right)^{2}$. Note that $\left(\left[x_{1}\right]-\left[x_{2}\right]\right)^{2}=[0,1]=\left\{f\left(x_{1}, x_{2}\right), x_{1} \in\left[x_{1}\right], x_{2} \in\right.$ $\left.\left[x_{2}\right]\right\} \subset[f]_{N}([0,1],[0,1])$. This illustrates the dependency problem which is a major concern in interval arithmetic. $f$ has multiple occurrences of variables that are handled as different variables by interval arithmetic. In general, the dependency problem implies an overestimation of the interval image. It renders NP-hard the problem of finding the optimal interval image of a polynomial [8]. This raises the need to symbolic manipulations of expression before calculations so as to reduce overestimation.

The PolyBox algorithm presented in this paper aims at solving nonlinear systems of constraints or Numerical CSPs. An NCSP $P=(V, C,[V])$ contains a set of constraints $C$, a set $V$ of $n$ variables with domains $[V] \in \mathbb{R}^{n}$. A solution $S \in[V]$ to $P$ satisfies all the constraints in $C$. To approximate all the solutions of an NCSP with interval-based techniques, the solving process starts from an initial box representing the search space and builds a search tree, following a Branch $\& 3$ Contract scheme. A Branching operation bisects the current box on 
one dimension (variable), generating two sub-boxes. At each node of the search tree, contraction/filtering algorithms improve the bounds of the current box with no loss of solutions. The process terminates with boxes of size smaller than a given positive $\omega$.

The constraint programming community proposes constraint propagation algorithms that perform a propagation loop like AC3. Contracting optimally a box w.r.t. an individual constraint is referred to as hull-consistency problem. Similarly to the optimal interval image computation, due to the dependency problem, hull-consistency is not tractable. The main procedure of our algorithm is compared to two state-of-the-art revise algorithms that handle the constraints individually. HC4-Revise [2] is known to achieve the hull-consistency of constraints having no variable with multiple occurrences, provided that the function ${ }^{1}$ is continuous. It traverses twice the tree representing the mathematical expression of the constraint for narrowing all the involved variable intervals. BoxNarrow $[2,14]$ is stronger than HC4-Revise [4] and can enforce hull-consistency of a constraint when it contains one variable with multiple occurrences. In the general case, it enforces the Box-consistency property [2].

Definition 2 An $\operatorname{NCSP}(X, C,[X])$ is box-consistent if every pair $(c, x), c \in$ $C, x \in X$ is box-consistent. Consider the pair $(c, x)$, where the constraint is described by $c: f\left(x, y_{1}, \ldots, y_{k-1}\right)=0, f: \mathbb{R}^{k} \rightarrow \mathbb{R}$, and the univariate interval function $f_{[Y]}(x)=f\left(x,\left[y_{1}\right], \ldots,\left[y_{k-1}\right]\right)$. The pair $(c, x)$ is box-consistent (with respect to the natural extension $\left.[f]_{N}\right)$ on the domain $[x]=[\underline{x}, \bar{x}]$, if:

$0 \in\left[f_{[Y]}\right]_{N}([\underline{x},+])$ and $0 \in\left[f_{[Y]}\right]_{N}([-, \bar{x}])$, where $[\underline{x},+]$ and $[-, \bar{x}]$ denote intervals of size one u.l.p. ${ }^{2}$ at the bounds of $[x]$.

In practice, for every pair $(f, x)$, starting with an interval $[x]$, the BoxNarrow procedure returns a reduced interval $\left[x^{\prime}\right] \subseteq[x]$ such that $\left[\underline{x^{\prime}},+\right]\left(\right.$ resp. $\left.\left[-, \overline{x^{\prime}}\right]\right)$ is the smallest (resp. largest) $\epsilon$-solution ${ }^{3}$ of the equation $f_{[Y]}(x)=0$. Existing procedures use a shaving principle to narrow $[x]$ : "Slices" $[\underline{x}, \underline{x}+\eta]($ resp. $[\bar{x}-\eta, \bar{x}])$ are discarded from $[x]$ if $0 \notin\left[f_{[Y]}\right]_{N}([\underline{x}, \underline{x}+\eta])\left(\operatorname{resp} .0 \notin\left[f_{[Y]}\right]_{N}([\bar{x}-\eta, \bar{x}])\right)$. This test sometimes uses a uni-variate interval Newton procedure.

Figure 1 illustrates that $f_{[Y]}$ is an interval function. It also shows the steps followed by BoxNarrow. The top (resp. the bottom) side of the figure details the "dichotomic" work performed by LeftNarrow (resp. RightNarrow) on slices/intervals of decreasing size, starting from $[x]$. For LeftNarrow, if the size of the current interval $[l]$ is less than or equal to 1 u.l.p. and $0 \in\left[f_{[Y]}\right]_{N}([l])$, then the procedure returns $[l]$. The last step 17 replaces the interval $[x]$ by the new interval $[\underline{l}, \bar{r}]$. Observe that at the end of RightNarrow (step 16), $[r]$ does not contain any zero of the function but an $\epsilon$-zero. The slicing performed by BoxNarrow on a variable $x$ limits the overestimation effect on $x$, but not on the other variables $y_{i}$ if they also occur several times.

\footnotetext{
1 along with projection functions used during the second top-down tree traversal...

2 One Unit in the Last Place is the gap between two successive floating-point numbers.

${ }^{3} x \in \mathbb{R}^{n}$ is an $\epsilon$-solution of $f(x)=0$, if $[-\epsilon, \epsilon] \cap f(x) \neq \emptyset$.
} 


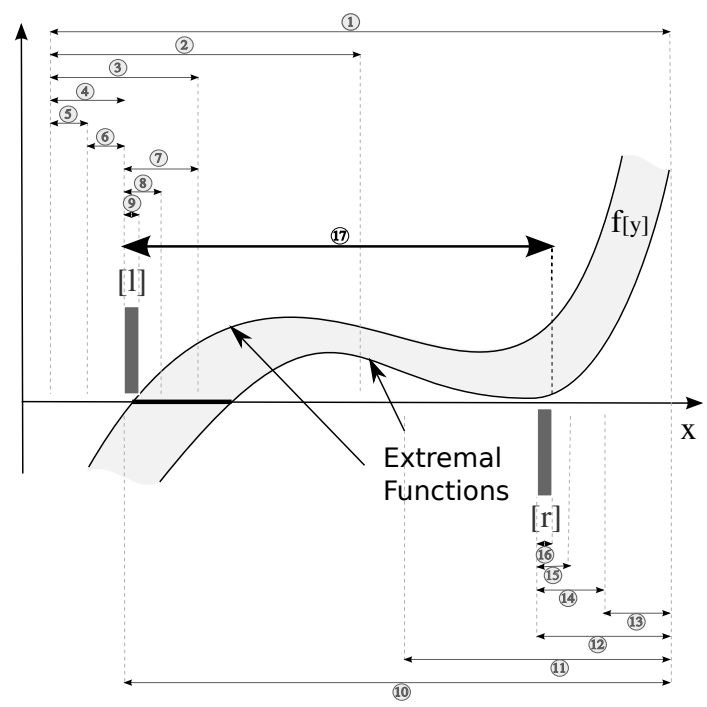

Fig. 1. The BoxNarrow procedure. The algorithm returns the interval computed in the step 17. (The additional contraction performed by Newton is not considered.)

\section{Description of the PolyBoxRevise procedure}

The aim of our new PolyBoxRevise procedure is to limit the overestimation due to multiple occurrences of variables $y_{i}$ and to speed up the iterative narrowing process introduced above. Before solving the system, in a preprocessing phase, for every pair $(c, x)$ given by $c: f\left(x, y_{1}, \ldots, y_{k-1}\right)=0$, we use symbolic manipulation to rewrite $f$ into a new form $g\left(x, y_{1}, \ldots, y_{k-1}\right)$. This preprocessing allows the PolyBoxRevise procedure to rapidly extract, during constraint propagation, non-interval extremal functions that enclose $g_{[Y]}$, before contracting $[x]$.

\section{Symbolic manipulation and extremal functions}

For any pair $(c, x)$ and any box $\left([x],\left[y_{1}\right], \ldots,\left[y_{k-1}\right]\right)$, the aim would be to extract the optimal extremal real (i.e., non-interval) functions $\underline{h}(x)$ and $\bar{h}(x)$ defined by $\underline{h}(x)=\min _{y_{i} \in\left[y_{i}\right]} f\left(x, y_{1}, \ldots, y_{k-1}\right)$ and $\bar{h}(x)=\max _{y_{i} \in\left[y_{i}\right]} f\left(x, y_{1}, \ldots, y_{k-1}\right)$, $\forall x \in[x]$. Then a necessary and sufficient condition for $x \in[x]$ to satisfy $c$ is $0 \in[\underline{h}(x), \bar{h}(x)]$. Unfortunately, it is generally not tractable to determine $\underline{h}$ and $\bar{h}$ due to the overestimation implied by the dependency problem (see Section 2 and [8]), and we have to be satisfied with functions $\underline{g}$ and $\bar{g}$ such that for any $x \in[x]$ we have $\underline{g}(x) \leq \underline{h}(x) \leq f\left(x, y_{1}, \ldots, y_{k-1}\right) \leq \bar{h}(x) \leq \bar{g}(x), \forall x \in[x], \forall y_{i} \in$ $\left[y_{i}\right], i \in\{1, \ldots, \bar{k}-1\}$. Now the test $0 \in[g(x), \bar{g}(x)]$ is only a necessary condition. We will refer to $\underline{g}$ and $\bar{g}$ as minimal and maximal extremal functions.

The aim is to automatically and rapidly identify extremal functions. This can clearly not be done for any function $f$ and we have restricted our attention to the class of functions that can be described by 


$$
f\left(x, y_{1}, \ldots, y_{k-1}\right)=\sum_{i=0}^{d} f_{i}\left(y_{1}, \ldots, y_{k-1}\right) \cdot h_{i}(x)
$$

where $d$ is a positive integer and $h_{i}$ has a finite number of zeros in $[x]$ that can be computed exactly. In addition, the sign of $h_{i}(x)$ is known for any $x \in[x]$. We have in mind elementary functions such as $x^{i}, \log (x)$ or $e^{x}$.

We have used the symbolic manipulation tool Mathematica [15] for automatically identifying functions $f_{i}$ and $h_{i}$, and to rewrite them in the most appropriate manner. The procedure FullSimplify of Mathematica computes automatically several possible forms for every $f_{i}$ (heuristically) and selects the form minimizing a given criterion. The criterion we have specified is the number of occurrences of each variable $y_{i}$. During the solving, like BoxNarrow, the PolyBoxRevise procedure first replaces, in the new analytic form $g$, the variables $y_{i}$ by their domains. We thus obtain $g_{[Y]}(x)=\sum_{i=0}^{d}\left[f_{i}\right]_{N}([Y]) h_{i}(x), \quad[Y]=\left(\left[y_{1}\right], \ldots,\left[y_{k-1}\right]\right)$. Given the box $[Y]$, the coefficients $\left[f_{i}\right]_{N}([Y])$, denoted $\left[c_{i}\right]$, are now numerical intervals. Due to the assumptions on $h_{i}$, the following two functions $g_{[Y]}$ and $\overline{g_{[Y]}}$ are respectively minimal and maximal extremal functions, computed at the bounds of the interval coefficients $\left[c_{i}\right]$ :

$$
\begin{gathered}
\underline{g_{[Y]}}(x)=\sum_{i=1}^{d} c_{i}^{-}(x) h_{i}(x) \quad \text { and } \quad \overline{g_{[Y]}}(x)=\sum_{i=1}^{d} c_{i}^{+}(x) h_{i}(x) \\
\text { with }\left\{\begin{array}{lll}
c_{i}^{-}(x)=c_{i}, & c_{i}^{+}(x)=\overline{c_{i}}, & \text { if } h_{i}(x) \geq 0 \\
c_{i}^{-}(x)=\overline{c_{i}}, & c_{i}^{+}(x)=\underline{c_{i}}, & \text { if } h_{i}(x) \leq 0
\end{array}\right.
\end{gathered}
$$

Example. Consider the function: $f\left(x, y_{1}, y_{2}\right)=\left(y_{1}+y_{2}\right) x^{2}+\left(2 y_{1} y_{2}\right) x+\sin \left(y_{2}\right)$. For the domains $\left[y_{1}\right]=[0.5,1],\left[y_{2}\right]=[1,2]$, we have $g_{[Y]}(x)=[1.5,3] x^{2}+[1,4] x+[0.84147,1]$ and then

$$
\begin{aligned}
& \underline{g_{[0.5,1],[1,2]}}(x)=\left\{\begin{array}{l}
1.5 x^{2}+x+0.84147, \text { if } x \geq 0 \\
1.5 x^{2}+4 x+0.84147, \text { if } x \leq 0
\end{array}\right. \\
& \overline{g_{[0.5,1],[1,2]}}(x)=\left\{\begin{array}{l}
3 x^{2}+4 x+1, \text { if } x \geq 0 \\
3 x^{2}+x+1, \text { if } x \leq 0
\end{array}\right.
\end{aligned}
$$

Remark. $g_{[Y]}$ and $\overline{g_{[Y]}}$ are optimal extremal functions of $g_{[Y]}$. However, although $f$ and $g$ are the same, the interval functions $f_{[Y]}$ and $g_{[Y]}$ are different because the replacement of the variables $y_{i}$ by $\left[y_{i}\right]$, occurring several times in $f$, produce different overestimations. Hence, $g_{[Y]}$ and $\overline{g_{[Y]}}$ constitute only approximate noninterval functions enclosing $f$. Also, Box-consistencies of $f_{[Y]}$ and $g_{[Y]}$ are not comparable. That is why our contractor starts by calling systematically the cheap HC4-Revise procedure on the initial form $f$ before performing the process described below.

For a given $[Y]$, once the extremal functions have been determined, we proceed with the contraction part of PolyBoxRevise (during constraint propagation). Starting with an initial interval $[x]$, let us detail how the new and improved left bound $\underline{l}$ ([l] is 1 u.l.p. large) of $[x]$ is determined. (A symetric process is performed for the right bound.) PolyBoxRevise first determines with which extremal function to work with. Three cases occur: 
1. If $g_{[Y]}(\underline{x}) \leq 0$ and $0 \leq \overline{g_{[Y]}}(\underline{x}): l=\underline{x} \quad$ (no contraction)

2. If $\overline{g_{[Y]}}(\underline{x})>0: g_{[Y]}$ is selected

3. If $\overline{\overline{g_{[Y]}}}(\underline{x})<0: \quad \overline{g_{[Y]}}$ is selected (situation depicted in the left side of Fig. 1)

The smallest root $[l]$ of $\overline{g_{[Y]}}(x)=0$ in $[x]$ can now be computed using the standard BoxNarrow (i.e., LeftNarrow) procedure applied to the extremal function selected. The advantage is a faster convergence since BoxNarrow is run with a non-interval function.

We have implemented the polynomial case, where $h_{i}(x)=x^{i}, i=0, \ldots, d$. In particular, when the degree $d$ is smaller than 4, instead of using BoxNarrow to determine the real roots of $\overline{g_{[Y]}}(x)=0$, we have used explicit analytical expressions of the roots. For $d=3$, we have used the Cardano's expressions ${ }^{4}$ of the real roots. We have adapted these symbolic methods to manage rounding errors due to floating point calculation by first replacing all the coefficients by a degenerate interval (of null size).

Finally, we have implemented a new procedure PolyBoxRevise based on the Box algorithm variant called BC4 [2]. If $f\left(x, y_{1}, \ldots, y_{k-1}\right)$ has a single occurrence of $x$, PolyBoxRevise calls HC4-Revise (like BC4 does). Otherwise, it uses the rewritten form $g$ (with appropriate symbolic expressions for the $f_{i}\left(y_{1}, \ldots, y_{k-1}\right)$ ) of $f$ produced automatically by the FullSimplify procedure of Mathematica [15] in the preprocessing. Four cases occur:

1. $f$ is not polynomial w.r.t. $x$ : the procedure calls BoxNarrow (or HC4-Revise in a hybrid version because it is less time consuming).

2. $g_{[Y]}(x)$ contains only one occurrence of $x$ : HC4-Revise is applied to $g_{[Y]}(x)$.

3. $g_{[Y]}(x)$ has multiple occurrences of $x$ and $d<4$ : analytic determination of the smallest root of $g_{[Y]}(x)$ in $[x]$.

4. $g_{[Y]}(x)$ has multiple occurrences of $x$ and $d \geq 4$ : numerical determination of the smallest root of $g_{[Y]}(x)$ in $[x]$, using BoxNarrow.

Remarks. The second case above can be illustrated by an equation of the system Caprasse (tested below): $-2 x+2 t x y-z+y^{2} z=0$ that our symbolic tool rewrites into: $(-2+2 t y) x+(-1+y 2) z=0$ (for the contraction of $[x]$ or $[z]$ ). Observe that the new form makes disappear the multiple occurrences of $x$ and $z$. The decrease in occurrences of $x$ and $z$ illustrates a successful transformation leading to a gain in CPU time. An equation of the instance 6body shows a counterproductive transformation of $5\left(b_{1}-d_{1}\right)+3\left(b_{2}-d_{2}\right)\left(b_{1}+d_{1}-2 f_{1}\right)=0$ into $b_{1}\left(5+3\left(b_{2}-d_{2}\right)\right)+\left(-5+3 b_{2}-3 d_{2}\right) d_{1}+6\left(-b_{2}+d_{2}\right) f_{1}=0$. Indeed, for obtaining an expanded form on $b_{1}$ or $d_{1}$, the transformation increases the overestimation because of the additional occurrences of variables $a_{2}, b_{2}$ and $d_{2}$.

\section{Comparison with the Box algorithm of Numerica}

Van Hentenryck, Michel et Deville have also used extremal functions (without using this vocabulary) in their interval-based solver Numerica [14]. The principle

\footnotetext{
${ }^{4}$ G. Cardano. Ars magna, sive de regulis algebraicis liber unus, Nuremberg, 1545.
} 
is introduced in one page in a technical article [13]. Numerica manages different forms of the handled system, and a separate constraint propagation is run on the system in an entirely expanded form for using extremal functions.

PolyBox follows on the contrary a scheme close to BC4. It manages a unique system with revise procedures adapted to every pair $(f, x)$, which causes an overestimation smaller than the entirely expanded form used by Numerica. In addition, like BC4, PolyBox also uses HC4-Revise when $x$ occurs only once in $f$. Finally, the analytic solving of low degree polynomials is added.

\section{Experiments}

Table 1. Results. The entries in the last four columns are the CPU time in second (first row) and the number of nodes in the search tree (second row).

\begin{tabular}{|l|r|r||c|c||c|c|}
\hline Name & \#var & \#sol & HC4 & BC4 & PolyBox-- & PolyBox \\
\hline Caprasse & 4 & 18 & 5.53 & 37.2 & 2.34 & $\mathbf{2 . 1 6}$ \\
& & & 9539 & 6509 & 2939 & 2939 \\
\hline Yamamura1 & 8 & 7 & 34.3 & 13.4 & 5.79 & $\mathbf{2 . 7 2}$ \\
& & & 42383 & 4041 & 2231 & 2231 \\
\hline Extended Wood & 4 & 3 & $\mathbf{0 . 7 6}$ & 1.94 & 1.34 & 1.12 \\
& & & 4555 & 1947 & 3479 & 3479 \\
\hline Broyden Banded & 20 & 1 & $>3600$ & 0.62 & 0.16 & $\mathbf{0 . 0 9}$ \\
& & & $?$ & 1 & 1 & 1 \\
\hline Extended Freudenstein & 20 & 1 & $>3600$ & 0.19 & 0.22 & $\mathbf{0 . 1 1}$ \\
& & & $?$ & 121 & 121 & 121 \\
\hline 6body & 6 & 5 & $\mathbf{0 . 5 8}$ & 2.93 & 0.73 & 0.73 \\
& & & 4899 & 4797 & 4887 & 4887 \\
\hline Rose & 3 & 18 & $>3600$ & $>3600$ & $\mathbf{4 . 0 0}$ & $\mathbf{4 . 1 0}$ \\
& & & $?$ & $?$ & 12521 & 12521 \\
\hline Discrete Boundary & 39 & 1 & 179 & 29.5 & 41.8 & $\mathbf{1 6 . 1}$ \\
& & & 185,617 & 3279 & 3281 & 3281 \\
\hline Katsura & 12 & 7 & $\mathbf{1 0 2}$ & 404 & $\mathbf{1 0 3}$ & $\mathbf{1 0 4}$ \\
& & & 14007 & 11371 & 13719 & 13719 \\
\hline Eco9 & 8 & 16 & $\mathbf{6 6}$ & 191 & 71 & 71 \\
& & & 132,873 & 125,675 & 131,911 & 131,911 \\
\hline Broyden Tridiagonal & 20 & 2 & 470 & 495 & 403 & $\mathbf{3 5 0}$ \\
& & & 269,773 & 163,787 & 164,445 & 164,445 \\
\hline Geneig & 6 & 10 & 3657 & $>7200$ & 3508 & $\mathbf{3 3 6 3}$ \\
& & & $79,472,328$ & $?$ & $4,907,705$ & $4,907,705$ \\
\hline
\end{tabular}

We have compared our PolyBox algorithm to BC4 and HC4. The symbolic manipulation of all pairs $(f, x)$ is achieved in a fraction of a second in a preprocessing by Mathematica [15]. All the contractors have been implemented in the free Ibex interval-based C++ library [3]. To find all the solutions to the tested 
NCSPs, the solving strategy bisects the variables in a round-robin way. Between two branching points in the search, constraint propagation (i.e., PolyBox, HC4 or BC4) is performed before an interval Newton.

Among the 44 polynomial systems with isolated solutions found in COPRIN's Web page ${ }^{5}$, we have selected the 12 instances that are solved by at least one of the 3 strategies in a time comprised between 1 second and 1 hour (on a Pentium $3 \mathrm{GHz}$ ) and have equations with multiple occurrences of the variables.

Table 1 reports interesting speedups brought by PolyBox on these instances. The column PolyBox-- in the table corresponds to a variant of PolyBox in which the low degree polynomials are not handled analytically but by BoxNarrow. The additional gain brought by the analytic process is significant in only two NCSPs.

Our first results are promising, so that it should be worthwhile hybridizing PolyBox with other algorithms, especially those achieving the Box-consistency or a weaker form of it $[1,5]$. An idea would be to keep the rewritten forms only if they are of degrees 2 and 3, and add them as global and redundant constraints in the system for improving the constraint propagation.

\section{References}

1. I. Araya, G. Trombettoni, and B. Neveu. Making Adaptive an Interval Constraint Propagation Algorithm Exploiting Monotonicity. In Proc. CP, LNCS, 2010.

2. F. Benhamou, F. Goualard, L. Granvilliers, and J.-F. Puget. Revising Hull and Box Consistency. In Proc. ICLP, conf. on logic programming, pages 230-244, 1999.

3. G. Chabert. Ibex - An Interval Based EXplorer. www.ibex-lib.org, 2010.

4. H. Collavizza, F. Delobel, and M. Rueher. Extending Consistent Domains of Numeric CSP. In Proc. IJCAI, pages 406-413, 1999.

5. A. Goldsztejn and F. Goualard. Box Consistency through Adaptive Shaving. In Proc. ACM SAC, pages 2049-2054, 2010.

6. L. Jaulin. Interval Constraint Propagation with Application to Bounded-error Estimation. Automatica, 36:1547-1552, 2000.

7. R. B. Kearfott. Rigorous Global Search: Continuous Problems. Kluwer, 1996.

8. V. Kreinovich, A.V. Lakeyev, J. Rohn, and P.T. Kahl. Computational Complexity and Feasibility of Data Processing and Interval Computations. Kluwer, 1997.

9. J.-P. Merlet. Interval Analysis and Robotics. In Symp. of Robotics Research, 2007.

10. R. E. Moore. Interval Analysis. Prentice-Hall, Englewood Cliffs N.J., 1966.

11. M. Rueher, A. Goldsztejn, Y. Lebbah, and C. Michel. Capabilities of Constraint Programming in Rigorous Global Optimization. In NOLTA, 2008.

12. W. Tucker. A Rigorous ODE Solver and Smale's 14th Problem. Found. Comput. Math., 2:53-117, 2002.

13. P. Van Hentenryck, D. McAllester, and D. Kapur. Solving Polynomial Systems Using a Branch and Prune Approach. SIAM J. on Num. Analysis, 34(2), 1997.

14. P. Van Hentenryck, L. Michel, and Y. Deville. Numerica : A Modeling Language for Global Optimization. MIT Press, 1997.

15. S. Wolfram. Mathematica. Cambridge University Press, fourth edition, 1999.

\footnotetext{
${ }^{5}$ See www-sop.inria.fr/coprin/logiciels/ALIAS/Benches/benches.html. These benchmarks have been proposed by the interval community and some of them correspond to real problems.
} 